\title{
DESIGN AND DEVELOPMENT OF A GIS-BASED PLATFORM USING OPEN SOURCE COMPONENTS FOR MONITORING, MAINTENANCE AND MANAGEMENT OF ROAD NETWORK: THE CASE STUDY OF CYPRUS
}

\author{
G. Christou ${ }^{1}$, A. Georgiou, ${ }^{1,}$, E. Christodoulou ${ }^{1}$, M. Tziakouri ${ }^{1}$, C. Christodoulou ${ }^{1}$, S. Kasinopoulou ${ }^{1}$, C. G. Panayiotou ${ }^{1}$, A. Savva $^{2}$ \\ ${ }^{1}$ University of Cyprus / KIOS Research and Innovation Center of Excellence, Cyprus (christou.george@ucy.ac.cy; georgiou.m.andreas@ucy.ac.cy; \\ christodoulou.eirini@ucy.ac.cy; tziakouri.maria@ucy.ac.cy; christodoulou.chrystalleni@ucy.ac.cy; kassinopoulou.stefani@ucy.ac.cy; \\ christosp@ucy.ac.cy) \\ ${ }^{2}$ Public Works Department, Ministry of Transport, Communications and Works, Cyprus (asavva@pwd.mcw.gov.cy)
}

KEY WORDS: Road Network, Transportation, Public Sector, Mobile Application, Platform, Open-Source, GIS, QGIS, Cyprus.

\begin{abstract}
:
Road transport is an important infrastructure system that has a vital role in economic progress, sustainable development, and urban prosperity of societies. Thus, geospatial fundamentals of transport systems need to be adequately considered by the organizations that manage such infrastructures. Furthermore, the adoption of methodologies for monitoring, control and optimisation of transportation networks, from a geographical perspective is crucial to deploy or utilize resources efficiently and cost-effectively. This paper aims to describe the implementation of a complete Geographical Information (GI) based system as developed for the needs of the Cyprus road transport infrastructure. The paper focuses on the system architecture and the presentation of the desktop and mobile application interfaces and utilities. The GI-based system provides a good example of how different tools and open-source components can be integrated to provide a complete solution for collecting, storing, managing, analysing and disseminating geographical information data related to road network infrastructure. The use of the platform within the organization has resulted in a noticeable increase in productivity and accountability. The errors in data collection, analysis and storage were minimized and thus the quality of available data been significantly improved. This is a step towards the achievement of the goals of the European Digital Strategy by using Geographical Information System technologies to take reliable and accurate decisions related to road network monitoring, maintenance and management.
\end{abstract}

\section{INTRODUCTION}

Transportation plays a vital role in the economic progress, sustainable development and urban prosperity of societies all over the world (Agyemang, 2013). Geographical Information Systems (GIS) technologies, consist of a fundamental tool for transportation (GIS-T), especially for applications related to route planning, maintenance, asset management and decision support and thus to the development of Intelligent Transport Systems (ITS) (Seguí Pons and Ruiz Pérez, 2003). ITS, rely on accurate data and well-performing communication, management and analysis components, each with a distinct spatial notion (Loidl et al., 2016).

The integration of multiple functionalities within one environment has cause organisations related to transportation to adopt GIS technologies within their workflow. Furthermore, the adoption of methodologies for monitoring transportation networks or optimisation of maintaining transport-related infrastructure, from a geographical perspective is crucial to deploy or utilize resources efficiently and cost-effectively.

Regarding the growing integration of geospatial functionalities and transportation, the main objective of this paper is to describe a complete GI-based system as developed for the needs of the public sector (main operator of the road infrastructure of Cyprus). The proposed framework comprises a combination of opensource components for the design and implementation of the platform. The rest of the paper is organized as follows. Section 2 is devoted to the description of the system architecture and the methodological approach. A presentation and illustration of the user interface (UI) for both desktop and mobile application are given in Section 3 and in the closing Section 4, concluding remarks as well future work aspects are discussed.

\subsection{Background}

The University of Cyprus, through the KIOS Research and Innovation Centre of Excellence (KIOS $\mathrm{CoE}$ ), in cooperation with the Public Works Department (PWD) of the Ministry of Transport, Communications and Works of Cyprus, is undertaking research activities on ITS. Under this perspective, an integrated GI-based platform with the acronym "GNOSIS" (Geographic INformatiOn System for Intelligent Transport System) is developed to collect, store, analyse, manage and disseminate data regarding the local transport network. The UI of the developed platform consists of three main components: Desktop application, Web Portal and a cross-platform Mobile application.

\section{METHODS}

\subsection{Development Methodology}

For the development of an information system, a methodology approach must be formalized to implement the system development life cycle (SDLC). There are many different system development methodologies and they vary in terms of the progression that is followed through the phases of the SDLC (Kendall and Kendall, 2006). "GNOSIS" platform was based on a hybrid agile-waterfall methodology, integrating that way the best elements of both models into the software development practices (Figure 1).

The waterfall development methodology was used for the system specifications analysis, where analysts and users proceeded sequentially from one phase to the next. The key deliverables for each phase were presented and approved. This phase consisted of the requirement collection, analysis and system design which was used for the design of the overall system architecture. The main advantage of this methodology is the identification of the system

\footnotetext{
*Corresponding author: georgiou.m.andreas@ucy.ac.cy
} 
requirements before programming and limiting changes as the project proceeds (Kendall and Kendall, 2006).

The main functions of the system were then split and the Agile development methodology was used for their development. This approach is a group of programming-centric methodologies that focus on streamlining the SDLC. For each sub-function of the project, face-to-face communication was undertaken involving programmers and users to ensure the usability of each developed tool or function. This was an iterative process in which every iteration was a complete software package which was then fitted to the main system. That includes requirements analysis, architecture planning and design, coding and development as well testing and documentation. Cycles kept short with the developing team focusing on adapting to the current business environment (Kendall and Kendall, 2006; Qumer and HendersonSellers, 2008).

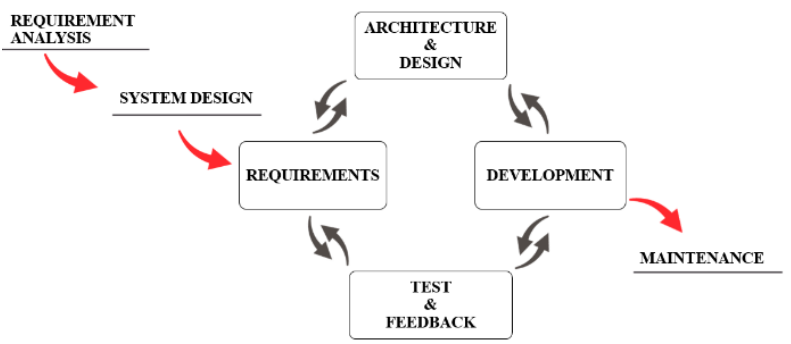

Figure 1. Hybrid agile-waterfall methodology approach.

\subsection{Basic Notions on Standardization}

The methodological framework for the system development was based on the ISO/IEC/IEEE 42010:2011 International Standard, which addresses the creation, analysis, and sustainment of architectures of systems using architecture descriptions. The standard provides a core ontology for the description of software architectures and proposes a conceptual model of architecture description, including concepts such as views, models, concerns, rationale, viewpoints, frameworks and architecture description languages (Standard, 2011).

In addition, the ISO/IEC/IEEE 29148:2018 International Standard was also used as a guide to identify the processes and products related to the requirements collection for the system throughout the life cycle of the project. This standard defines the structure of the requirement collection, the required functionalities and characteristics of the system as well the iterative and recursive application of requirement processes needed (Standard, 2018).

\subsection{System Architecture Model}

The system architecture model involves a coherence of several subsystems. Figure 2 provides a schematic diagram of the proposed system architecture with the operational functions and interactions among them. Level 1 , refers to all external data sources that were configured, transformed, and loaded to the database server which is based on object-relational database system of PostgreSQL with the extension PostGIS (Level 2). Level 3 refers to the Application Server, which communicates the data to the end-user via the Front End of the desktop application, web portal and mobile application. The GeoServer was introduced to manage the data stored in the spatial database and to provide the final image visualized to the end-user. In addition, the Django web framework was utilized for the webserver backend development and for the authentication service. Level 4 refers to user identification and management (IAM) from which access is given to the respective users (Level 5). Finally, on Level 6 , all the three components of the developed system are grouped.

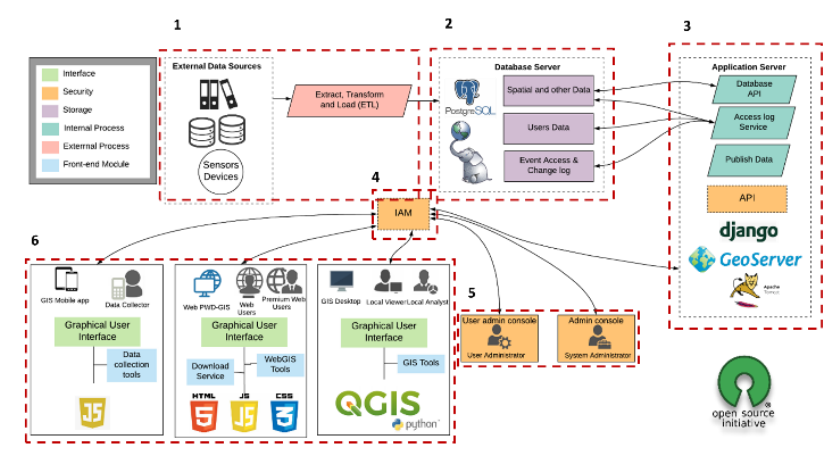

Figure 2. Subsystems schematic diagram.

\subsection{Sub-systems Description}

\subsubsection{Database Server}

The geodatabase consists of a dataset collection of spatial data and related attribute tables of the transport network of Cyprus. The fundamental datasets in the geodatabase are feature classes, raster datasets and tables. All data are harmonized, stored and transformed via services for publication under the INSPIRE infrastructure (European Parliament, 2009). PostgreSQL has been chosen as an indispensable system component for management and PostGIS extension as the mean for storage of the geographic objects, for topological operations within PostgreSQL and maintains data integrity (Price et al., 2021). In effect, PostGIS spatially enables PostgreSQL to be used as a backend spatial database for GIS. This multiplatform has low operating costs, supports various data types and supports the Open Geospatial Consortium (OGC) standard with many desktop GIS clients (Alba et al., 2019). Moreover, the selection of PostGIS was based on its better performance compared to Oracle spatial, Mongo DB and Neo4J for selected object operations and spatio-temporal data queries (Makris et al., 2019; Sharma et al., 2018; Shukla et al., 2016).

Figure 3 presents a simplified example of the entity-relationship diagram, as designed based on INSPIRE infrastructure. The Network Element refers to every element of the road network which provides some function that is of interest in the network; the Network Reference provides general properties to associate the network-related phenomena with the network elements, and; Network Property refers to a reference of a network property to a network element (European Parliament, 2009). 


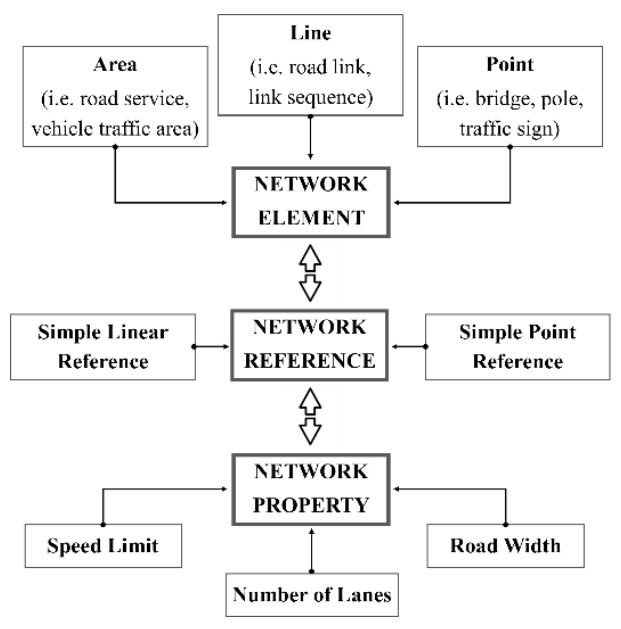

Figure 3. Simplified entity-relationship diagram.

\subsection{Application Server}

The application server communicates the data to the end-user via the front-end of the "GNOSIS" platform, through three interfaces: a desktop application, a web portal and a mobile application. The front-end is designed to allow the visualisation of various types of spatial data formats (e.g. GeoJSON, Web Map Service, Web Feature Service, ESRI Shapefile, GeoPackages, GeoTIFF) and 3D models with small size files. The application server comprised of the GeoServer and Django web framework. The GeoServer was introduced to manage the data stored in the spatial database and to provide the final image visualized by the end-user (Gobakis et al., 2017). GeoServer supports various types of spatial data format and implements the services required for the visualization. It also supports all interfaces and provides editing functionality for the geospatial data. Moreover, the GeoServer system used, based on the concepts of users' levels, user groups and roles, where the permissions of reading, writing, administration of data and services are determined by roles that are assigned to users or user groups.

The web development framework Django on the other hand has implemented by default security measures including support for PostgreSQL database with a straightforward query process through object-relational mapping (Alba et al., 2019). It has been used for the webserver backend and as a mean of authentication service.

\subsubsection{Communication System}

The communication system must comply with specific standards in order to ensure the interconnection of the components and technologies with the main platform (Gobakis et al., 2017). The communication protocol between the front-end and the end-user is performed via the Internet using secure transfer protocols. Through the IAM, access for both data and developed tools is given to various end-user categories with specific privileges for security purposes (Figure 4) as follows: System Administrator has access to the developed toolset and access to all available data from all settlements with the capability to modify the database. Also, the system administrator is responsible for system hardware and software respectively; the User Administrator has access to the developed toolset and access to all users' data from all settlements with the capability to modify the database. In addition, the user administrator has the responsibility to solve any access problems of the users, to add or remove them from the system and change their access level; the User Application Programmer has editing access on the developed toolset and access to all available data from all settlements with the capability to modify the database; the Local Analyst has access to all available data from all settlements and the capability to modify the departmental database and use the departmental tools; the Local User has only reading access to all available data and departmental tools, and; the Data Collector related to mobile application users for fieldwork. The responsibility of this user is limited to data collection, database upgrade with the collected data and read access to the rest database.

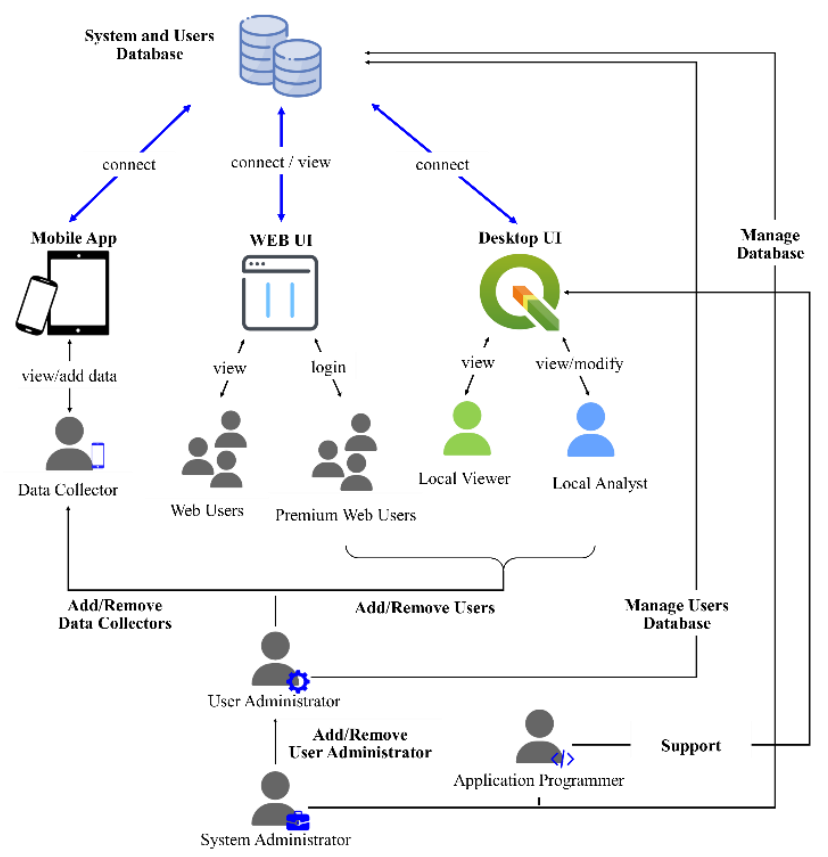

Figure 4. End-user categories of the system.

\subsubsection{User Interfaces}

The most important functionalities of 'GNOSIS' platform relies on a Desktop version and a Mobile application respectively. Both were designed, planned and implemented according to the needs of PWD's employees.

The Desktop version is developed to store, analyse, manage, and disseminate data regarding the local transport network. QGIS open source software licenced under GNU (a collection of free software; the acronym stands for "GNU's not Unix") General Public License (GPL), was chosen as a highly suitable platform for the development and application of the desktop version. QGIS integrates with other open sources GIS packages and provides a Python application program interface, which is used to expand its functionality and efficiently exploit the capabilities of a GIS.

The Mobile application was developed for data collection on the field using smart mobile devices. The mobile app is integrated with the desktop version in the terms of data view, task management and follow-up. The open-source Ionic Framework was adopted as an emerging approach to proffer solutions to the cross-platform mobile app development problems. An integration with angular application design framework also utilized to provide several components to achieve interactive mobile UI and response media queries (Dunka et al., 2017). Angular was primarily used to manage the application's logic and data. The hybrid approach on the development, allows a single code base work for different platforms (Windows and iOS). 


\section{RESULTS AND BENEFITS}

\subsection{Desktop Application}

The desktop UI (Figure 5), is based on the open source software QGIS and is divided into panels for each of its core functions of the system such as map canvas, map navigation, layers and tools. The 'GNOSIS' menu has been added where all the developed functionalities were grouped to easily be accessible by the authorized users.

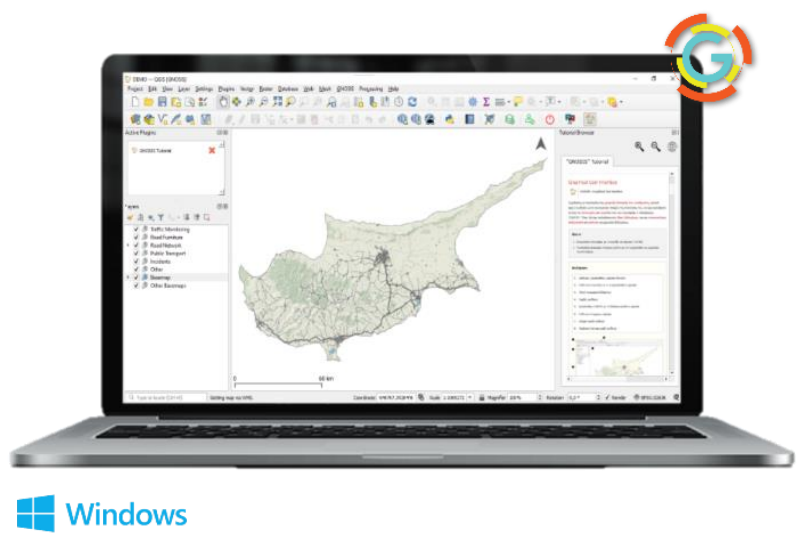

Figure 5. Desktop User Interface.

More specific, 100 specialized functions - QGIS plug-ins (Figure 6) were developed to assist the everyday workflow of PWD employees, covering a wide range of applications for storing, analysing, managing and disseminating data (spatial or not spatial) relevant to the local transport network. Moreover, through the desktop version, the user can prepare reports and thematic maps through pre-set layouts and access to a volume of over 10GB of data with more than 50 unique datasets. In addition, the desktop version integrates efficiently with the mobile application for assigning tasks and monitoring their progress for PWD field working teams.

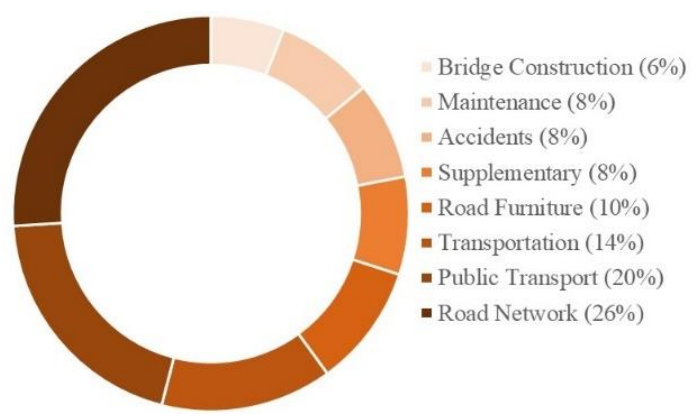

Figure 6. Allocation of developed specialized functionalities.

The use of the desktop version of 'GNOSIS' platform along with the use of embedded QGIS analysis tools on the everyday workflow of the employees, has proven a noticeable increase on the productivity and accountability on a departmental level. The errors in data collection, analysis and storage were minimized and thus the quality of produced data was significantly improved.

\subsection{Mobile Application}

The mobile application that developed provided smart solutions and tools for the needs of fieldwork. Through the UI (Figure 7), users can access their everyday tasks and navigate through the map to identify the respect spatial allocation. The status of the task can be changed (on progress, on hold or finished) according to the completion level and be monitored by the supervisor through the desktop version. Any data relevant to static information of the road network (e.g., traffic signs) or the condition of the road network can be collected and stored to database. Thus, any information related to the above can be examined and analysed locally from the system administrator, assigning new tasks and proceed with all necessary actions for the road maintenance.
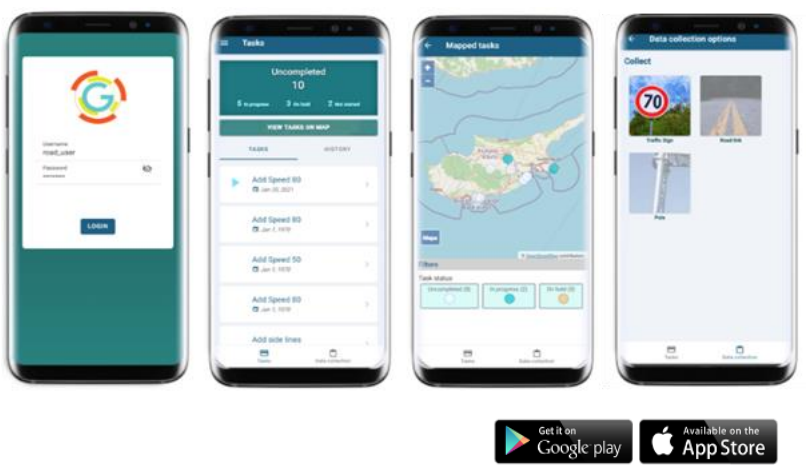

Figure 7. Mobile application User Interface screens

\section{DISCUSSION AND CONCLUDING REMARKS}

The objective of this paper was to demonstrate how open source tools may effectively support the monitoring, optimization, and maintenance of a transport-related infrastructure by the development of a completed GI-based system.

The major findings can be summarized in some key points. First, the developed system consists of a good example of how different tools and open-source components can be integrated, providing a complete solution for collecting, storing, managing, analysing and disseminate geographical information data. Open-source software provides benefits equivalent to those provided by commercial products, such as the low cost of ownership, more frequent software and functionality updates and most importantly, the ability to extensively customize the software to meet the needs of the organization or the user itself. Another key point is that the implementation of all the data in a single spatial database linked to a dynamic use and representation of spatial information from the entire organization. From this standpoint, the dissemination of spatial information within PWD, improves the internal procedures on data usage, exploitation, data production, storage and thus data quality. Also, it provides opportunities for the users to understand the important role of geo-visualization, to harmonize a large amount of data, share knowledge about the spatial features regarding local road network and reduce the cooperation gap within the department. Moreover, the user-friendliness of both desktop version and mobile application is considered a strength as internal procedures and needs were made smooth and fast, increasing both productivity and accountability. For PWD, this is a step towards achieving their goals of the European Digital Strategy by using GIS technologies to take reliable and accurate decisions related to road network monitoring, maintenance, and management. A further key point is the integration of QGIS in the use of plugins for data pre-processing and post-processing within a customized environment. This implies no need for supplementary software for processing and analysing data, without entailing the need for costly licensing, while providing the chance for developing tailored applications. Finally, regarding the mobile application implementation, the Ionic framework proved to be an outstanding benefit in the terms of speed development and the cross-platform 
development application which can run on both Android and iOS environments.

Nevertheless, it must be highlighted that the success of the developed platform, relies on the employees of PWD commitment and involvement. Adopting the platform on the everyday workflow along with their support on training materials and workshops, the system will significantly grow within the organization.

\subsection{Future Recommendation}

Given the importance of further support and evolvement of the entire system, the following aspects should be taken into consideration.

A further improvement in performance for both desktop and mobile application is needed. The developed system can support new capabilities and functionalities, upgrading at the same time the overall performance and system reliability. Moreover, the concept of Big Data has been also examined, as new challenges appear in multi-processing, analysing, archiving, sharing, transferring, and processing large datasets across organizations in the public sector. According to previous research (Fredriksson et al., 2017) the public sector can significantly boost productivity by actively using Big Data as they can address many internal problems such as enhancing efficiency and productivity, transparency and well-being. Moving towards this concept, Internet of Things (IoT) should be introduced to the public sector. A network of physical devices embedded with the system, sensors, actuators and network connectivity will enable these objects to collect and exchange data, supporting various advanced trends such as comprehensive interconnection, transferable information and cloud-based applications (Tiwary et al., 2018; Zhang et al., 2019). IoT is expected to have great potential in terms of both economic and social values. Finally, an in-depth exploration of how artificial intelligence methods can be employed to solve and optimize complicated problems. Potential models can focus on the enhancement of the existing public transportation network, on the design of the expansion of the network or safety management (Kouziokas, 2017; Shatnawi et al., 2020).

\section{ACKNOWLEDGEMENTS}

This research was funded by the Public Works Department (PWD) of the Ministry of Transport, Communications and Works of Cyprus, under a research contract with the University of Cyprus, through the KIOS Research and Innovation Centre of Excellence (KIOS CoE) for the fiscal years 2017-2020.

The work of G. Christou, A. Georgiou, E. Christodoulou, M. Tziakouri, C. Christodoulou, S. Kasinopoulou and C.G. Panayiotou has been partially supported by the European Union's Horizon 2020 research and innovation program under grant agreement No 739551 (KIOS CoE) and by the Republic of Cyprus through the Deputy Ministry of Research, Innovation and Digital Policy.

The authors would also like to thank all the PWD employees for their valuable assistance, remarks and suggestions throughout the design, implementation and testing of the developed platform 'GNOSIS'.

\section{REFERENCES}

Agyemang, E., 2013. A cost-effective Geographic Information Systems for Transportation (GIS-T) application for traffic congestion analyses in the Developing World. Ghana J. Geogr. $5,51-72$.
Alba, C.E.G., Bastos, L.C.C., Cruz-Roa, A., Celis, C.A.D., 2019. Geographic Information System on the Web for recording georeferenced information transporting system at the Universidad de los Llanos Web GIS system for transportation of the Universidad de los Llanos. Proc. - 2019 7th Int. Conf. Softw. Eng. Res. Innov. CONISOFT 2019 164-172. https://doi.org/10.1109/CONISOFT.2019.00032

Dunka, B., Emmanuel, E.A., Oyerinde, Y., 2017. Hybrid Mobile Application Based on Ionic Framework Hybrid Mobile Application Based on Ionic Framework Technologies. Int. J. Recent Adv. Multidiscip. Res. 4, 3121-3130.

European Parliament, 2009. INSPIRE Data Specification on Transport Networks - Guidelines.

Fredriksson, C., Mubarak, F., Tuohimaa, M., Zhan, M., 2017. Big Data in the Public Sector: A Systematic Literature Review. Scandinavian Journal of Public Administrator. ISSN, 2001-7405. Gobakis, K., Mavrigiannaki, A., Kalaitzakis, K., Kolokotsa, D.D., 2017. Design and development of a Web based GIS platform for zero energy settlements monitoring. Energy Procedia 134, 48-60. https://doi.org/10.1016/j.egypro.2017.09.598

Kendall, K., Kendall., J., 2011. Systems Analysis and Design, $8^{\text {th }}$ Edition. Prentice Hall

Kouziokas, G.N., 2017. The application of artificial intelligence in public administration for forecasting high crime risk transportation areas in urban environment. Transp. Res. Procedia 24, 467-473. https://doi.org/10.1016/j.trpro.2017.05.083

Loidl, M., Wallentin, G., Cyganski, R., Graser, A., Scholz, J., Haslauer, E., 2016. GIS and transport modeling-strengthening the spatial perspective. ISPRS Int. J. Geo-Information 5. https://doi.org/10.3390/ijgi5060084

Makris, A., Tserpes, K., Spiliopoulos, G., Anagnostopoulos, D., 2019. Performance evaluation of MongoDB and PostgreSQL for spatio-temporal data. CEUR Workshop Proc. 2322.

Price, A., Langford, M., Higgs, G., 2021. Computing geographical access to services: The design of a client-server solution that incorporates multiple transport modes. Trans. GIS 1-19. https://doi.org/10.1111/tgis.12744

Qumer, A., Henderson-Sellers, B., 2008. A framework to support the evaluation, adoption and improvement of agile methods in practice. J. Syst. Softw. 81, 1899-1919. https://doi.org/10.1016/j.jss.2007.12.806

Seguí Pons, J.M., Ruiz Pérez, M., 2003. Geographic Information Systems and Intelligent Transport Systems : technologies used to form new communication networks. Netcom 17, 53-70. https://doi.org/10.3406/netco.2003.1572

Sharma, M., Sharma, V.D., Bundele, M.M., 2018. Performance Analysis of RDBMS and No SQL Databases: PostgreSQL, MongoDB and Neo4j. 3rd Int. Conf. Work. Recent Adv. Innov. $\begin{array}{llll}\text { Eng. } & \text { ICRAIE } & 2018 & 2018,\end{array}$ https://doi.org/10.1109/ICRAIE.2018.8710439

Shatnawi, N., Al-Omari, A.A., Al-Qudah, H., 2020 Optimization of Bus Stops Locations Using GIS Techniques and Artificial Intelligence. Procedia Manuf. 44, 52-59. https://doi.org/10.1016/j.promfg.2020.02.204 
Shukla, D., Shivnami, C., Shah, D., 2016. Comparing Oracle Spatial and Postgres PostGIS. Comput. Sci. Electron. J. 7, 95100. https://doi.org/10.090592/IJCSC.2016.116

Standard, I., 2011. INTERNATIONAL STANDARD ISO / IEC / IEEE 42010. Systems and software engineering - Architecture description.

Standard, I., 2018. INTERNATIONAL STANDARD ISO / IEC / IEEE 29148. Systems and software engineering - Life Cycle processes - Requirements engineering.

Tiwary, A., Mahato, M., Chidar, A., Chandrol, M.K., Shrivastava, M., Tripathi, M., 2018. Internet of Things (IoT): Research, Architectures and Applications. Int. J. Futur. Revolut. Comput. Sci. Commun. Eng. ISSN, 2454-4248.

Zhang, Y., Ma, X., Zhang, J., Hossain, M.S., Muhammad, G., Amin, S.U., 2019. Edge Intelligence in the Cognitive Internet of Things: Improving Sensitivity and Interactivity. IEEE Netw. 33, 58-64. https://doi.org/10.1109/MNET.2019.1800344 